\title{
A vaccinotherapia serve effectivamente no tratamento da blenorrhagia e suas complicações?
}

(De um opusculo do Dr. Fortunato Gino-Spina, director do "Ambulatorio Dermosiphilopathico" do Hospital de Suzzara).

o uso das vaccinas antigonococcicas no tratamento da blenorrhagia e das suas complicações foi recebido, no seu inicio, com grande enthusiasmo pela maioria dos medicos, porém, especialmente n'estes ultimos tempos, muitos enthusiasmos desvaneceram, e muitos foram os que, perdida toda a fé, abandonaram completamente o uso das vaccinas antigonococcicas, pois não julgaram util este tratamento e alguns, antes, chegaram a affirmar que o tratamento por meio das vaccinas antigonococcicas não é isento de perigos, sendo notado o insurgir de complicações depois de seu uso.

Não exito em affirmar com toda a certeza, haver um grande exagero n'isto tudo, e ter-se que attribuir, os casos nos quaes logo após o uso da vaccina antigonococcica appareceram complicações (orchi-epydidimites, prostatites etc.), ao facto que a complicação achava-se em caminho, digamos assim, quando recorremos á vaccina, e que a vaccina não poude, porque já tarde, prevenir a complicação.

Nos casos, nos quaes não tivemos uma acção manifestamente benefica, devemos attribuir este insuccesso ao facto de que hoje, quasi sempre a maior parte dos medicos julga que a blenorrhagia seja produzida exclusivamente pelo gonococco e por conseguinte, pretendemos cural-a com o emprego unicamente da vaccina gonococcica pura. (AIguns autores calculam na porcentagem de $25 \%$ as urethrites primitivas não gonococcicas).
ZIPPARI GAROLA com o uso da vaccina antigonococcica, juntamente com o tratamento local, observou que as complicações já existentes são atacadas e curadas immediatamente, sem deixar nenhum residuo; observou tambem que a acção da vaccina desenvolve-se prevalentemente no processo urethral agudo essencialmente de 2 maneiras; favorecendo a defeza por parte dos elementos da mucosa, e attingindo o gonococco seja na superficie como profundamente até nos lugares mais escondidos e que esta acção se explica igualmente bem. e rapidamente na blenorrhagia chronica e que a vaccina encurta muito o tempo do tratamento.

CHATELEIN tem curado alguns casos de complicações da blenorrhagia com a "Vaccina antigonoccica Bruschettini", e achou que esta vaccina dá resultados muito mais rapidos que as demais vaccinas.

Este facto seria devido a polimicrobicidade; polimicrobicidade a qual age não somente contra o gonococco mas tambem contra todos os microbios associados, e em particular contra o estaphylococco, que muitos autores agora julgam ter a maior importancia nas complicações da blenorrhagia e ao methodo de preparação do cultivo "in vivo", dos germens, que tornaria a vaccina mais rapidamente e mais facilmente usufruivel da parte do organismo.

LE FUR que se occupa muitissimo da questão presente, diz que para se obterem resultados evidentes da vaccinotherapia é necessario servir-se d'este methodo segundo as concepçôes bacteriologicas modernas: diz que existem numerosos casos de urethrites 
primitivas não gonococcicas, devidas em geral ao estaphylococco, ao enterococco, á união de diversos coccos; que mesmo nas urethrites gonococcicas o papel do gonococco é menos importante, que não se julgue á primeira vista.

Affirma, com effeito, que emquanto este papel é importante nos primeiros 8 a 15 dias da molestia, diminue pouco a pouco a medida que nos afastamos do inicio da mesma, tanto que no periodo sub-agudo e sobretudo chronico da blenorrhagia, o gonococco passa em segunda linha e torna-se tão negligenciavel que os microbios associados, os microbios secudarios e tambem os saprophytas, sobresahem, tornando-se seu papel mais importante do que o do gonococco, e occupanı sosinhos o primeiro plano da scena pathologica. Frequentemente, pois, acontece que o gonococco não pode ser encontrado, ou excepcionalmente, em muitas formas blenorrhagicas chronicas.

Para fortalecer quanto acima tem dicto, Le Fur apresentou, á Sociedade de Medicina de Paris, uma estatistica pessoal sobre 831 casos de secreções urethraes quaesquer e concluiu que:

em $33 \%$ dos casos foi encontrado o gonococco só; em $20 \%$ dos casos gonococco associado a outros microbios; em $48 \%$ microbios secundarios (enterococcos, diversos) sem nenhum gonococco, estaphylococco, pseudo-diphtericos, e em $10 \%$ a urethrite era aseptica ou melhor amicrobica.

Além na blenorrhagia aguda:

$87 \%$ com gonococco no estado de pureza.

$12 \%$ com gonococco associado a outros microbios.

Na blenorrhagia chronica:

$7 \%$ com gonococco no estado de pureza;

$8 \%$ com gonococco associado a outros microbios;

$72 \%$ com microbios secundarios sem nenhum gonococco (sobretudo estaphylococco e enterococco).

Nas complicações da blenorrhagia, ellas são mantidas quasi na totalidade dos casos por microbios associados e secundarios (particularmente estaphy- lococco) como sustentou Le Fur no Congresso de Urologia de Strasburgo em 1921 e como demonstrou Lavenant para as epididimites.

$$
* * *
$$

De quanto temos dicto é mais do que evidente a necessidade de examinar, toda a vez que se apresenta um paciente atacado por uma forma blenorrhagica, microscopicamente a secreç̧ão urethral, exame que nos deverá dizer com toda a clareza se nos encontramos em frente a uma urethrite mantida exclusivamente pelo gonococco ou com associação microbiana secundaria ou si se trata de uma urethrite não gonococcica.

Tambem nas, formas gonococcicas puras, depois do primeiro periodo de tratamento, será util e necessario examinar ainda a secrecção pois que está assegurado que a medida que nos afastamos do inicio da molestia temos quasi sempre a associação de microbios secundarios.

Por isso depois do uso da vaccina antigonococcica pura teremos que empregar a vaccina que será mais indicada pelo resultado do exame da secrecção, vaccina esta necessariamente polymicrobiana. (1).

Si recorrermos a um exame microscopico completo da secreção, as vaccinas polymicrobianas, isto é, as que contem as varias especies microbianas que podem interessar a molestia em acção, dão ás vezes grande resultado, sobretudo nas formas iniciaes e devem conter pelo menos: gonococcos, estaphilococcos, enterococos, pseudo-diphtericos, etc.

E' claro que contemporaneamente ao emprego da vaccinotherapia, temos que fazer tambem o tratamento local (lavagens, instillações, dilatações, massagens etc.) porque, como bem diz Lefur, as vaccinas servem especialmente a combater a infecção, e o tratamento local evita ou faz desapparecerem as lesões.

(1) Usar n'este caso, a Vaccina Antigonococcica alternada com a do Antipiogeno Polyvalente Bruschettini. 
Tive occasião de empregar o NeoDmegon em alguns doentes e de chegar á conclusão que o preparado correspondia bem especialmente nas complicações da blenorrhagia, publicando pois uma serie de casos nos quaes foi empregado o dito preparado.

Como n'esta publicaçãơ eu não admittia a acção da vaccina Bruschettini superior ao Neo-Dmegon, sobretudo nas formas simples de urethrites blenorrhagicas (Lavatelli) bem que nunca a tivesse usado, quiz, devido a conselho do Prof. Bruschettini, controlar a affirmação de Lavatelli.

As minhas experiencias foram feitas em doentes que se apresentaram alguns no meu consultorio particular de Mantua e outros no Ambulatorio Dermosyphilopathico annexo ao Hospital Civil de Suzzara.

Os doentes foram divididos em:

a) atacados por blenorrhagia aguda;

b) por blenorrhagia chronica;

c) por complicações blenorrhagicas.

Technica, intervallo, doses e numero das injecções - Empreguei a via endomuscular. Geralmente as injecções foram feitas em dias alternados ou cada dois dias. Quasi sempre comecei com 1 c. c. passando a $1 \mathrm{~cm} .1 / 2$, até $2 \mathrm{~cm}$. cubicos. 0 numero de injecções variou conforme a edade do mal.

Reacção geral ou local das injecções - Ambas foram quasi sempre nullas ou minimas.

$\Lambda$ reacção geral (na primiera ou segunda injecção) póde consistir em um pequeno augmento de temperatura e a um mau estar geral; a local raramente apparece, na primeira injecção e só consecutivamente, podemos ter leve dor.

A's vezes podemos observar, depois das primeiras injecções, um augmento da secrecção urethral, e nas formas complicadas, um augmento, muito passageiro, da inflammação na séde da complicação (reacção dos fócos).

\section{Resultados obtidos na blenorrhagia aguda.}

Convenci-me de que nas formas iniciaes de urethrite gonococcica a aç̧ão da vaccina antigonococcica Bruschettini é de uma efficacia muito grande tanto que eu a aconselho como sendo necessaria para poder antes de tudo, (acção preventiva) impedir as complicações, secundariamente porque a secrecção urethral fica beneficamente influenciada tanto que, a não ser algum caso em que encontramos a assim chamada phase negativa, com augmento da secrecção na $1 .^{\mathrm{a}}$ e $2 .^{\mathrm{a}}$ injecção, diminue rapidamente e com ella tambem o gonococco desapparece.

Os phnomenos dolorosos param rapidamente $\mathrm{e}$ as urinas não são mais turvas como no inicio.

Nas urethrites posteriores agudas com tenesmo vesical, stranguria, com emissões de sangue no fim da micção, poucas injecções de vaccina attenuam o mal e dão por conseguinte uma rapida cura.

\section{Resultado obtidos na blenorrhagia chronica.}

A vaccina demonstrou-se muitissimo util, pois que se viram casos em que a molestia permanecia apezar de todo tratamento, tambem mechanico, ceder, brilhantemente associando o tratamento vaccinico. Tivemos uma acção resolutiva e antibactericida muito marcada, sempre porque, n'estas formas chronicas, como já dissemos, além do gonococco temos sempre associados tambem outros germes que sentem um beneficio da polymicrobicidade da vaccina antigonococcica Bruschettini.

Nas formas acompanhadas de prostatites chronicas, os resultados foram ainda mais evidentes, encurtando muitissimo o tempo do tratamento, que é associado ao tratamento local com massagens, e de suppositorios resolventes.

\section{Resultados obtidos nas complicações da blenorrhagia}

Os resultados foram maravilhosos seja pelo effeito como pela rapidez. A complicação que mais sentiu os beneficios da vaccinotherapia foi a orchiepididimite simples ou dupla e com fol- 
liculite. O tratamento poude ser feito quasi sempre ambulatoriamente e se o doente tinha uma occupação sedentaria ou pelo menos não sujeita a excessivos esforços, tambem sem interromper o trabalho habitual.

Fiz sempre as injecções endomusculares nos gluteos e localmente uncções com pomada ao ichtyol com gayacol ou com pomada mercurial com belladona.

Nas outras formas complicadas (periurethrites, cooperites, vesiculites prostatites, bartolinites, salpingo-ovarites, etc.) obtivemos optimas resoluções e muitas vezes a vaccina resolveu prostatites agudissimas com ameaça de suppuração.

As reacções foram minimas e bem supportadas pelo doente que com vontade se prestava ao tratamento com a vaccina antigonococcica Bruschettini porque era e sentia as vantagens e o valor da mesma.

Alguns autores empregaram a vaccina antigonococcica Bruschettini com fim diagnostico, isto é, recorreram á possibilidade de pôr em evidencia o gonococco nos processos blenorrhagicos latentes, praticando um numero variado (de 6 a 12) de injecções de vaccina antigonococcica.

Sobre isto tenho uma unica observação na qual effectivamente o doente, que tinha tido uma blenorrhagia havia 10 annos e que não tinha mais apparecido apezar do pouco tratamento feito no tempo da molestia, achandose em vespera de casar, queria uma certeza formal.

Em lugar de recorrer aos meios usuaes (nitrato de prata, coito, e cerveja) fiz o tratamento de 8 injecções de vaccina antigonococcica Bruschettini, após as quaes foi feito o exame cultural das secrecções. Consegui assim demonstrar a presença do gonococco que, em bôa companhia tinha-se aninhado em recessos glandulares uretraes e prostaticos; prova que antes sem a vaccina antigonococcica tinha resultado negativo.

\section{Conclusões}

Posso, depois de quanto disse acima e melhor ainda depois dos evidentes resultados obtidos com o emprego da vaccina antigonococcica Bruschettini affirmar que:
A acção da Vaccina Antigonococcica Bruschettini - (vaccina polymicrobiana contendo varias raças de gonococco, pseudo gonococco, estaphilococco, pseudo dipheterico, enterococco, etc.) na blenorrhagia, aguda, subaguda e chronica é clara e dá sempre uma rapida acção attenuante dos phenomenos inflammatorios e uma rapida resolução dos infiltrados e localizações profundas da blenorrhagia chronica, quando este tratamento fôr associado ao local (lavagens, instillações, dilatações, massagens, etc.).

As complicações da blenorrhagia (especialmente orchi-epididimites, prostatites, cistites, etc.) são attenuadas e vencidas rapidamente, permittindo, na maioria dos casos, ao doente o tratamento ambulatorio e ao mesmo tempo o trabalho diario, com a condição que tenha cuidados.

Poude emfim observar que a vaccina não somente é efficaz no tratamento das localizações, diremos assim, secundarias da blenorrhagia (arthrites, epididimites, etc.) contra as quaes affecções as outras vaccinas tinham dado, precedentemente, resultados bons, mas que acima de tudo, explica uma acção curativa rapida e efficaz, nas urethrites blenorrhagicas, onde até agora os resultados da vaccinotherapia, tinham sido contradictorios e incertos.

Creio que isto não seja pouco: uma arma assim efficaz pode, antes deve ser, usada com a maxima confiança; necessariamente porém, devemos ter presentes as observações das quaes fallamos antes, para não ter a lamentar insuccessos que são, na grande maioria dos casos, causados por um mau uso da vaccina mesma.

São expostos os seguintes casos tratados e curados com a vaccina antigonococcica Bruschettini:

Urethrites blenorrhagica aguda $\begin{array}{r}\text { Casos } \\ 18\end{array}$

Urethrites blenorrhagica chronica. 17

Urethrites blenorrhagica subaguda. 6

Salpingo ovarites 2

Bartolinites 2

Folliculites 1

Urethrites e Cistites blenorrhagicas j

Ovaro-salpingite aguda 1

Cistite blenorrhagica 2

Metrite blenorrhagica 1

Urethrite total aguda $\quad 2$ 